Cite this: Phys. Chem. Chem. Phys., 2011, 13, 12268-12276

\title{
An infrared study of solid glycine in environments of astrophysical relevance
}

\author{
Belén Maté,* Yamilet Rodriguez-Lazcano, Óscar Gálvez, Isabel Tanarro and \\ Rafael Escribano
}

Received 24th March 2011, Accepted 6th May 2011

DOI: $10.1039 / \mathrm{c1cp20899c}$

The conversion from neutral to zwitterionic glycine is studied using infrared spectroscopy from the point of view of the interactions of this molecule with polar (water) and non-polar $\left(\mathrm{CO}_{2}, \mathrm{CH}_{4}\right)$ surroundings. Such environments could be found on astronomical or astrophysical matter. The samples are prepared by vapour-deposition on a cold substrate $(25 \mathrm{~K})$, and then heated up to sublimation temperatures of the co-deposited species. At $25 \mathrm{~K}$, the neutral species is favoured over the zwitterionic form in non-polar environments, whereas for pure glycine, or in glycine/water mixtures, the dominant species is the latter. The conversion is easily followed by the weakening of two infrared bands in the mid-IR region, associated to the neutral structure. Theoretical calculations are performed on crystalline glycine and on molecular glycine, both isolated and surrounded by water. Spectra predicted from these calculations are in reasonable agreement with the experimental spectra, and provide a basis to the assignments. Different spectral features are suggested as probes for the presence of glycine in astrophysical media, depending on its form (neutral or zwitterionic), their temperature and composition.

\section{Introduction}

The identification of interstellar organic molecules is a subject of great interest, as they may provide important insights into the history of the solar system and the origin of life on Earth. Amino acids have been found in Solar System bodies and meteorites, ${ }^{1-3}$ and in particular, cometary grains collected by the NASA Stardust mission ${ }^{4,5}$ have been shown to contain glycine, ${ }^{6}\left(\mathrm{NH}_{2} \mathrm{CH}_{2} \mathrm{COOH}\right)$, the simplest of the amino acids and the most vastly studied and searched. Glycine was also found in the analysis of the Almahata Sitta Meteorite, ${ }^{7}$ and although the authors could not discard some terrestrial contamination, the racemic composition of alanine in the meteorite indicated an alien origin of these amino acids. However, this subject is controversial to a high degree; see for instance the recent publication claiming fossils in meteorites ${ }^{8}$ and the heated discussion that it is generating.

In the laboratory, it has been demonstrated that glycine can be formed from ultraviolet irradiation of icy interstellar analogs, ${ }^{9-14}$ in the midst of a complex assortment of chemical species generated in the reactions induced by the irradiation. Although recent works have extended the spectroscopic range of study of amino acids even up to the vacuum ultraviolet, ${ }^{15}$ infrared spectroscopy is a frequently used tool to study laboratory generated amino acids, ${ }^{16}$ as well as to derive much

Instituto de Estructura de la Materia, IEM-CSIC, Serrano 121-123, 28006 Madrid, Spain.E-mail: belen.mate@csic.es of the available information on astrophysical ices, either from space or terrestrial observatories. The extension of laboratory infrared studies of amino acid molecules in different icy environments seems therefore of evident usefulness.

Glycine has the particularity that in water solution or in crystalline phases $(\alpha, \beta$ or $\gamma)$ it adopts a zwitterionic form $\left({ }^{+} \mathrm{NH}_{3} \mathrm{CH}_{2} \mathrm{COO}^{-}\right)$, whereas in the gas phase or in lowtemperature inert matrices it exists in its neutral form. ${ }^{17-19}$ The disposition of the amino and the carboxyl groups in the neutral species gives rise to three possible molecular structures that were early investigated by matrix-isolation by Stepanian et al. ${ }^{18}$ These authors also applied theoretical techniques to study their relative stabilities, a subject dealt later by Pacios et al. ${ }^{20}$ Acid and alkaline forms of glycine, with $\mathrm{Cl}^{-}$and $\mathrm{Na}^{+}$, respectively, were also studied by Rosado et al..$^{21}$ The association of glycine with water has given rise to recent studies of different types, using matrix-isolation Fourier transform infrared (FTIR) spectroscopy of glycine-water complexes, ${ }^{22}$ or focussed on the adsorption of glycine molecules on ice surfaces, ${ }^{23,24}$ and also of a theoretical nature, either by density functional theory (DFT) methods, ${ }^{22}$ or using molecular dynamics. ${ }^{25}$ Closer to the interest of this work is the thorough investigation by Gómez-Zavaglia and Fausto ${ }^{26}$ of the existence and interconversion of the neutral and zwitterionic forms for glycine and two methyl derivatives, using FTIR spectroscopy. They generated pure glycine solids by depositing neutral glycine vapour, sublimated in an oven at 87 or $64{ }^{\circ} \mathrm{C}$, on a very cold substrate $(9 \mathrm{~K})$, observing the neutral form of glycine 
in the solid state. They also noted that the proportion of neutral and zwitterionic forms in the solid depends on both the generation conditions of the vapour and the temperature of the deposition substrate. Upon annealing the amorphous neutral form irreversibly transforms into the zwitterionic one. The transformation can be followed by the evolving IR spectrum, which in these conditions is similar to the spectrum of crystalline $\alpha$-glycine.

Whereas glycine has thus received considerable attention both as a solid and in water solution, mixed species with other components, like $\mathrm{CO}_{2}$ or $\mathrm{CH}_{4}$, have not been studied yet, as far as we know. In astronomical or astrophysical media, such mixtures can be expected to occur, and this provides one of the reasons to undertake the present investigation.

We have generated low temperature solid binary mixtures (from $25 \mathrm{~K}$ to $200 \mathrm{~K}$ ) of glycine with water, carbon dioxide or methane containing about $0.5 \%$ of glycine, a proportion that is still probably high for astrophysical objects, but could be taken as a model for laboratory work. The mixed ice films have been studied by Fourier Transform Infrared spectroscopy. Our research is complemented by theoretical calculations and predictions of the IR spectra of solid crystalline glycine and of the gas phase glycine monomer and dimer.

\section{Experimental and computational methods}

\section{Experimental setup}

The experimental setup was described in detail elsewhere. ${ }^{27,28}$ In brief, it consists of a high vacuum chamber provided with a closed cycle helium cryostat, with an infrared transparent $\mathrm{Si}$ substrate in contact with the cryostat cold finger. The finger can be cooled down to $14 \mathrm{~K}$, although we have chosen $25 \mathrm{~K}$ for the present experiments. The residual pressure of the chamber at room temperature is $5 \times 10^{-8} \mathrm{mbar}$. Through adequate windows, the system is coupled to a Bruker Vertex70 FTIR spectrometer to work in transmission configuration. The main difference to our previous setup is the present capability of rotating the cryostat under vacuum, which has been used as described below.

Glycine salt was purchased from Sigma-Aldrich (purity $\geq 99.0 \%$ ). Low temperature solid layers were prepared by deposition of its sublimated vapour on the cold $\mathrm{Si}$ substrate. To evaporate the glycine a specifically designed sublimation mini-oven located inside the vacuum chamber was constructed. It consists of a copper crucible provided with a $50 \mathrm{~W}$ halogen lamp for heating and a chromel-alumel (tipe K) thermocouple for temperature measurements. The oven temperature is regulated by means of a proportional-integral-derivative (PID) controller. While the set-point temperature was being gained the deposition substrate was maintained tangential to the mini-oven flow. In this position the substrate holder intercepts the glycine flow, an effect that was confirmed by recording IR spectra during the oven heating process (see the scheme in Fig. 1). When the oven reached the working temperature, usually after 30 minutes of operation, the substrate was rotated and placed perpendicular to the glycine flow, and the oven was translated to position its exit hole, of $4 \mathrm{~mm}$ diameter, at $10 \mathrm{~mm}$ from the cold substrate. To finish the deposit the heating lamp was switched off and the

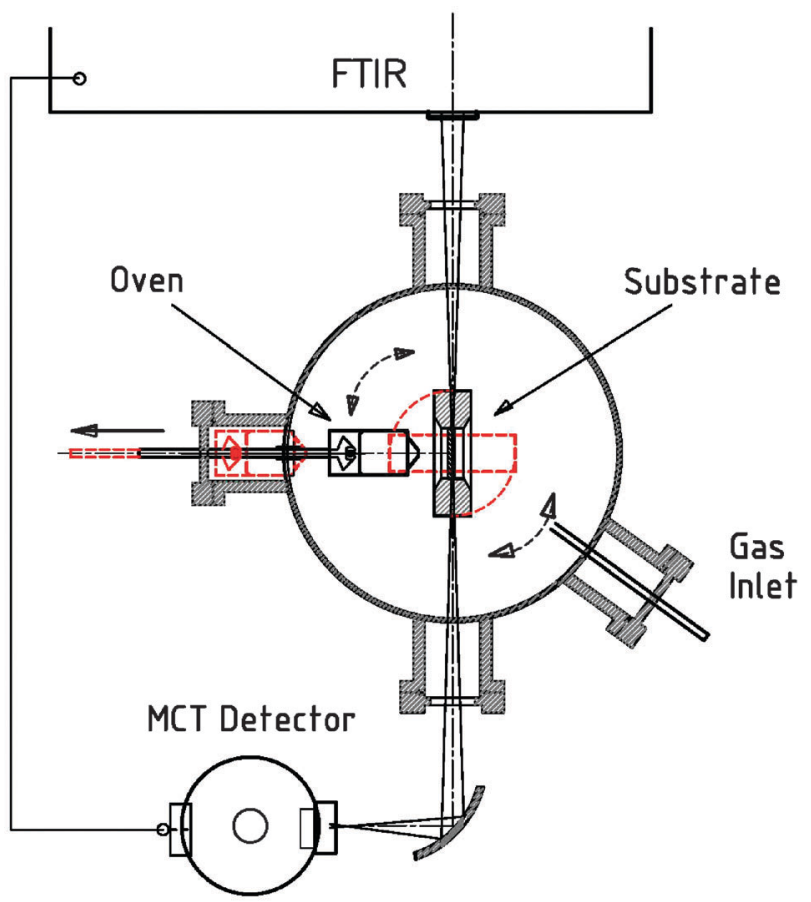

Fig. 1 Schematic representation of the cryostat, including the oven set-up.

oven was moved back to a place in contact with a copper block that accelerated its cooling.

The oven working temperature was selected to yield a high proportion of neutral glycine at $25 \mathrm{~K}$. According to Gómez-Zavaglia and Fausto ${ }^{26}$ the neutral to zwitterionic ratio in the deposit decreases with increasing oven temperature. After the initial study on the oven temperature dependence of the deposited samples described in the following section, we fixed the oven temperature at $105{ }^{\circ} \mathrm{C}$ for the rest of the experiments described in this work (Oven temperatures are indicated in Centigrade to keep a closer reference to the actual measuring employed and to avoid confusion with cryostat temperatures, expressed in Kelvin as customary). Mixed layers of glycine and the other components were prepared by introducing water vapour, $\mathrm{CO}_{2}$ or $\mathrm{CH}_{4}$ through an independent inlet to backfill the chamber to a controlled pressure of about $1 \times 10^{-5} \mathrm{mbar}$ at the same time that the glycine flow was being admitted. In this way a mixed glycine/ice layer was formed on one side of the substrate and a pure $\mathrm{H}_{2} \mathrm{O}, \mathrm{CO}_{2}$ or $\mathrm{CH}_{4}$ ice layer on the other side. In all the spectra discussed in this work the contribution of the pure species was removed by subtracting a properly scaled spectrum of the appropriate species recorded under the same experimental conditions. All spectra were recorded at $2 \mathrm{~cm}^{-1}$ resolution. The temperature evolution of the different spectra was followed by heating the solid deposits generated at $25 \mathrm{~K}$ with a $5 \mathrm{~K} \mathrm{~min}^{-1}$ ramp up to $200 \mathrm{~K}$.

Column densities of water, $\mathrm{CO}_{2}$ and $\mathrm{CH}_{4}$ in the ice mixtures were calculated via the Lambert-Beer relation, using the integrated values of the infrared absorption bands and the corresponding integrated absorption coefficients, $A$. The bands chosen for this purpose were the $\nu_{1}$ band of water around $3.1 \mu \mathrm{m}\left(3200 \mathrm{~cm}^{-1}\right)$, the $\nu_{3}$ band of $\mathrm{CO}_{2}$ at $\sim 4.3 \mu \mathrm{m}$ $\left(2340 \mathrm{~cm}^{-1}\right)$, and the $\nu_{4}$ band of $\mathrm{CH}_{4}$ at $\sim 7.7 \mu \mathrm{m}\left(1300 \mathrm{~cm}^{-1}\right)$. 
Reference values were taken from ref. 29 for water, from ref. 30 for $\mathrm{CO}_{2}$, and from ref. 31 for $\mathrm{CH}_{4}$. We used $A=1.9 \times$ $10^{-16} \mathrm{~cm}$ molecule ${ }^{-1}$ for water, $A=7.1 \times 10^{-17} \mathrm{~cm} \mathrm{molecule}^{-1}$ for $\mathrm{CO}_{2}$, and $A=8.085 \times 10^{-18} \mathrm{~cm} \mathrm{molecule}^{-1}$ for pure $\mathrm{CH}_{4}$ ice (corrected assuming a $\mathrm{CH}_{4}$ density of $0.47 \mathrm{~g} \mathrm{~cm}^{-3}$ as described by Herrero et l. $^{32}$ ). To determine glycine column densities we were faced with the problem of lacking experimental information on the integrated absorption coefficient of the glycine bands. We were then forced to use our calculated values for solid $\alpha$-glycine. The calculated integrated band strength summing all infrared bands in the $2000-800 \mathrm{~cm}^{-1}$ spectral region was $1.6 \times 10^{-15} \mathrm{~cm}$ molecule ${ }^{-1}$. The measured integral of the spectra of glycine deposits in the same spectral region, obtained after heating our samples to $200 \mathrm{~K}$, was then compared with the calculated magnitude.

The fraction of the neutral form in pure glycine samples was estimated by the $1600 \mathrm{~cm}^{-1}$ zwitterionic band intensity ratio between measurements at $200 \mathrm{~K}$, where no neutral species remain, and those taken at $25 \mathrm{~K}$. This procedure assumes that the absorption coefficient of this band does not change with temperature. In the three mixtures studied the relative intensities of the different bands of the spectra were compared with those of the pure glycine deposit in order to estimate the neutral/ zwitterion fraction.

\section{Computational methods}

We have applied theoretical methods to study glycine as a solid and as an isolated molecule. For the first type, we have used the CASTEP package, ${ }^{33}$ a program based on a density functional theory (DFT) plane-wave pseudo-potential method that is specifically designed to calculate different properties of solids, like geometry optimization and prediction of infrared spectra. In order to choose an optimum parameter set to simulate crystalline $\alpha$-glycine, we have tested several correlationexchange functionals using different parameterizations: $\mathrm{PBE}^{34}$ and $\mathrm{PW} 91^{35}$ gradient-corrected functionals (GGA), and nonlocal potentials as PBE0 ${ }^{36}$ and B3LYP. ${ }^{37}$ Dispersion corrections in two different schemes (Tkatchenko and Scheffler ${ }^{38}$ and Grimme $^{39}$ ) have also been included in the gradient correction functionals. PBE and PW91 simulations gave considerably shorter hydrogen bond distances in the optimized structure than the experimental value, around $15-20 \%$, but the results turned out to be much more accurate when dispersion corrections were included. From these tests it follows that all methods (PBE, PW91, B3LYP, PBE0), when dispersion corrections are taken into account, give similar geometry values of the $\alpha$-glycine crystal. Moreover, a similar conclusion was found regarding the IR spectrum for those cases when it was calculated, namely PBE and PW91. Consequently, we will present our results for just the PBE functional, including dispersion correction in the Tkatchenko and Scheffler ${ }^{38}$ scheme. Convergence criteria of $1.0 \times 10^{-5} \mathrm{eV}$ atom $^{-1}$, $0.03 \mathrm{eV} \AA^{-1}, 0.001 \AA$ and $0.05 \mathrm{GPa}$ for energy, maximum force, maximum displacement and maximum stress, respectively, were selected. Norm-conserving pseudo-potentials with a plane wave energy cutoff of $750 \mathrm{eV}$ were also used. The calculations were performed in a $k$-point grid of the entire Brillouin zone generated using a $k$-point separation of $0.07 \AA^{-1}$.
On the other hand, we used the Gaussian 03 suite $^{40}$ to study the isolated glycine molecule, its dimer, and glycine in a water environment. We chose a moderately high level of theory, namely $\mathrm{B} 3 \mathrm{LYP} /$ aug-cc-pvTZ, which afforded predicting the vibrational spectra with a reasonable level of accuracy. ${ }^{20}$ To compute the glycine monomer in a water environment, we did the calculations in a polarizable continuum model (PCM) using the default parameterization included in the Gaussian code.

\section{Results and discussion}

\section{Pure glycine solid films}

The temperature of the sublimation oven affects the proportion of neutral to zwitterionic form on the glycine deposit at low temperature. It has been proposed that the transformation from the neutral to the zwitterionic form occurs in the solid phase and depends on the energy that the molecules have previously to their deposition on a substrate held at low temperatures. $^{26}$

In Fig. 2a we present spectra of pure glycine deposits obtained changing the temperature of the oven and maintaining the substrate at $25 \mathrm{~K}$. Obviously the number of molecules evaporated, and hence the number of molecules in the sample increases with temperature. Therefore, to facilitate the

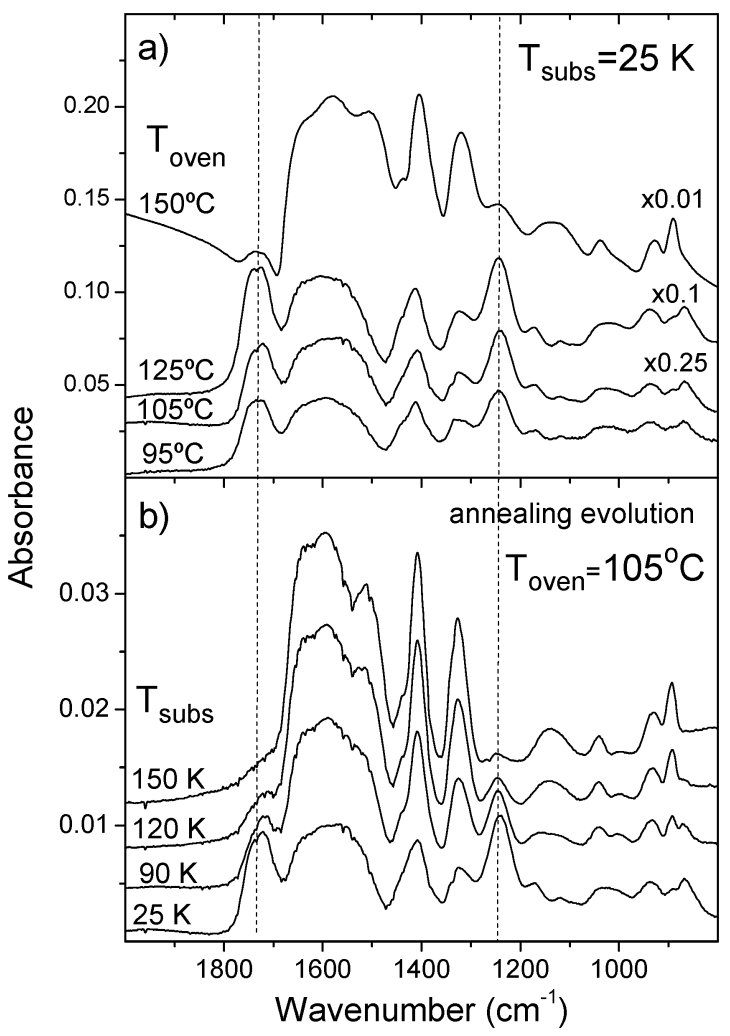

Fig. 2 (a) Spectra of glycine layers vapour-deposited at $25 \mathrm{~K}$ using different sublimation oven temperatures, indicated in the figure. (b) Spectra of a glycine layer generated at $T_{\text {oven }}=105{ }^{\circ} \mathrm{C}$, vapourdeposited at $T_{\text {subs }}=25 \mathrm{~K}$, and warmed to the indicated substrate temperatures. In both cases, the evolution of the neutral/zwitterionic ratio in the sample can be followed by changes on the main neutral glycine bands, marked by dashed vertical lines. Spectra are offset in the absorbance scale for clarity. 
comparison, the three upper spectra in Fig. 2a corresponding to oven temperatures of $150{ }^{\circ} \mathrm{C}, 125^{\circ} \mathrm{C}$ and $105{ }^{\circ} \mathrm{C}$ have been scaled down in intensity by factors of $0.01,0.1$ and 0.25 , respectively. The two strongest bands associated to neutral glycine in the spectral range represented in Fig. 2 appear at $1730 \mathrm{~cm}^{-1}$ and $1240 \mathrm{~cm}^{-1}$ (marked with dashed lines in the figure), whereas the zwitterionic species is the main component, but not necessarily the only one, of the other bands in this zone. When the oven temperature is between $95^{\circ} \mathrm{C}$ and $125^{\circ} \mathrm{C}$ a nearly constant neutral to zwitterionic ratio is observed in the deposit. However, at $150{ }^{\circ} \mathrm{C}$, the bands of the neutral species appear considerably weaker than those of the zwitterion. This behaviour was already observed by Gómez-Zavaglia and Fausto ${ }^{26}$ and it has been confirmed in our experiments, although at quite different oven temperatures. The explanation given by those authors is based on local heating effects. When hot glycine molecules hit the cold substrate their thermal energy can be used to induce proton transfer and thus convert the neutral species into the zwitterionic one, more stable in the solid phase. The higher the vapour temperature, the higher the induced local heating and, hence, a more efficient conversion from neutral to zwitterions is observed.

To complement this study, we represent in Fig. $2 \mathrm{~b}$ spectra recorded at increasing substrate temperatures of a sample generated at $T_{\text {oven }}=105{ }^{\circ} \mathrm{C}$ and deposited at $T_{\text {subs }}=25 \mathrm{~K}$, and subsequently warmed at $5 \mathrm{~K} \mathrm{~min}^{-1}$. The weakening of the main neutral glycine bands with growing temperature reveals the transformation of the neutral to the zwitterionic species. At $200 \mathrm{~K}$ the neutral species bands cannot be appreciated any more in the spectra. The spectrum at this temperature is shown in reference to a theoretical prediction later on in this work (see Fig. 4). If the sample is cooled back to $25 \mathrm{~K}$ the IR bands do not change appreciably.

The main parameters obtained from our DFT calculation of the zwitterionic $\alpha$-glycine crystal are shown in Table 1 , together with the experimental values measured by neutron diffraction, ${ }^{41}$ used as initial geometry in the optimization processes. A representation of the unit cell is shown in Fig. 3. As we mentioned above, dispersion corrections were necessary to improve the estimation of the cell parameters and H-bond distances, with the result that the calculated parameters are in acceptable agreement with the experimental measurements. In spite of these corrections, an expansion of the unit cell volume is obtained, particularly along the $b$-axis on which dispersive forces have a more relevant contribution. The calculations also predict $\sim 0.04 \AA$ shorter H-bond distances than those obtained by neutron powder diffraction. The theoretical results imply a greater proton transfer from the ammonium group to the $\mathrm{O}$ atom of a carboxyl group of a neighbouring molecule. These discrepancies in the evaluation of the dispersion forces and H-bonds are normally found in DFT methods (see for example ref. 42). Overall, for those parameters not involved in $\mathrm{H}$-bonding, our predicted intramolecular values are in good agreement with those experimentally obtained.

Based on the optimized structure, we have predicted the IR spectrum of the crystal, displayed in Fig. 4, and summarized in Table 2. As far as we know this is the first time that a calculated infrared spectrum of solid glycine is reported. The lower panel of the figure reproduces the experimental spectrum
Table 1 Calculated (this work) and experimental lattice parameters ( $a, b, c$ in $\AA, \beta$ in degrees, and volume in $\AA^{3}$ ) and bond distances $(\AA)$ of the $\alpha$-glycine crystal

\begin{tabular}{lcc}
\hline Parameter & Theoretical $^{a}$ & Experimental $^{b}$ \\
\hline$a$ & 5.128 & 5.105 \\
$b$ & 13.066 & 11.969 \\
$c$ & 5.481 & 5.465 \\
$\beta$ & 113.065 & 111.697 \\
Volume & 337.88 & 310.25 \\
$r(\mathrm{~N}-\mathrm{H})$ & 1.059 & 1.037 \\
$r(\mathrm{~N}-\mathrm{H})$ & 1.070 & 1.054 \\
$r(\mathrm{~N}-\mathrm{H})$ & 1.043 & 1.025 \\
$r(\mathrm{~N}-\mathrm{C})$ & 1.490 & 1.476 \\
$r(\mathrm{C}-\mathrm{H})$ & 1.098 & 1.090 \\
$r(\mathrm{C}-\mathrm{H})$ & 1.093 & 1.089 \\
$r(\mathrm{C}-\mathrm{C})$ & 1.531 & 1.526 \\
$r(\mathrm{C}-\mathrm{O})$ & 1.271 & 1.250 \\
$r(\mathrm{C}-\mathrm{O})$ & 1.260 & 1.251 \\
$r(\mathrm{NH} \cdots-\mathrm{O})_{\text {shortest }}$ & 1.688 & 1.728
\end{tabular}

${ }^{a}$ PBE gradient-corrected functional (GGA), with dispersion corrections in TS scheme. ${ }^{b}$ Ref. 41.

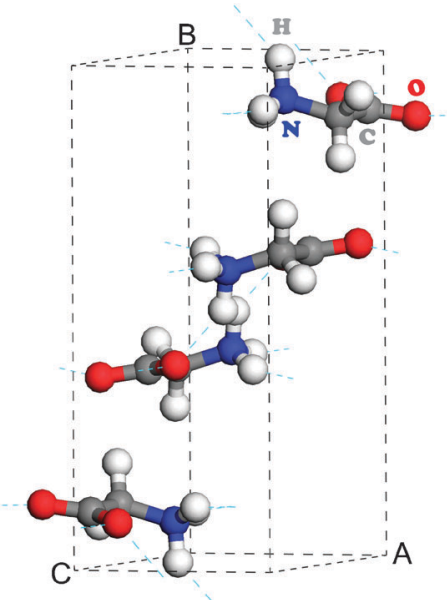

Fig. 3 Representation of the monoclinic unit cell of the calculated $\alpha$-glycine crystal.

of a pure glycine film obtained by deposition at $25 \mathrm{~K}$ and annealed to $200 \mathrm{~K}$. The comparison between the spectra is not straightforward, as the calculations do not provide information on bandwidths, an essential component in the appearance of any spectrum. Even so, the correspondence of the main absorptions between experiment and theory is clear, and the agreement could be made more evident on the overall relative intensities if the calculated bands were conveniently broadened. The deviations on the assigned vibrations collected in Table 2 are usually smaller than $5 \%$. We have not been able to find literature values for experimental IR band strengths of glycine. Thus, although the calculated intensities could have large uncertainties, we have used these values to estimate the number of glycine molecules in our deposits.

\section{Solid glycine in different environments}

We have generated mixed solid layers of glycine $/ \mathrm{H}_{2} \mathrm{O}$, glycine/ $\mathrm{CO}_{2}$ and glycine $/ \mathrm{CH}_{4}$ at $25 \mathrm{~K}$ with approximately $0.5 \%$ in mass glycine concentration. Fig. 5 displays the spectra of these 


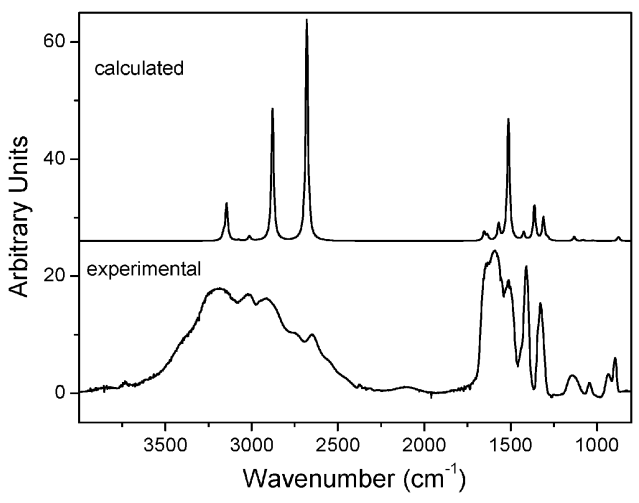

Fig. 4 IR calculated spectrum, as described in the Computational methods section, of solid crystalline $\alpha$-glycine (top) compared with a pure glycine ice film obtained by deposition at $25 \mathrm{~K}$ followed by heating at $200 \mathrm{~K}$. Theoretical bands are represented by Lorentzian curves with an arbitrary half-width at half-maximum of $8 \mathrm{~cm}^{-1}$.

Table 2 Spectral assignment of crystalline $\alpha$-glycine in the zwitterionic structure $^{a}$

\begin{tabular}{|c|c|c|c|c|}
\hline \multirow[b]{2}{*}{ Assignment } & \multicolumn{2}{|l|}{ Experimental } & \multicolumn{2}{|l|}{ Theoretical } \\
\hline & Wavenumber & Intensity & Wavenumber & Intensity \\
\hline$\nu \mathrm{NH}_{3}, \nu \mathrm{CH}_{2}$ & $3244-2551$ & vs & $\begin{array}{l}3144,3009 \\
2878,2680\end{array}$ & 17255 \\
\hline$\delta \mathrm{NH}_{3}$ & $1661-1512$ & $\mathrm{~s}$ & $1653-1512$ & 6503 \\
\hline$\delta \mathrm{CH}_{2}$ & 1436 & sh & 1424 & 331 \\
\hline$\nu \mathrm{COO}$ & 1407 & $\mathrm{~m}$ & 1361 & 1441 \\
\hline$\omega \mathrm{CH}_{2}, \omega \mathrm{NH}_{3}$ & 1326 & $\mathrm{~m}$ & 1309 & 989 \\
\hline$\rho \mathrm{NH}_{3}$ & 1138 & w & 1131 & 178 \\
\hline$\nu \mathrm{CN}$ & 1039 & $\mathrm{w}$ & 1078,1022 & 81 \\
\hline$\rho \mathrm{CH}_{2}$ & 929 & w & 878 & 51 \\
\hline$\nu \mathrm{CC}$ & 893 & w & 873 & 132 \\
\hline$\delta \mathrm{COO}$ & 671 & $\mathrm{w}$ & 676 & 314 \\
\hline
\end{tabular}

${ }^{a}$ Wavenumber values in $\mathrm{cm}^{-1}$ and theoretical intensities in $\mathrm{km} \mathrm{mol}^{-1}$. Theoretical spectra calculated as described in the Computational methods section. Intensities listed are addition of the corresponding individual values, where appropriate. Qualitative experimental intensities: vs, very strong, s, strong, sh, shoulder, m, medium, and w, weak.

diluted mixtures compared with one of the pure amino acids (top panel). In all cases the glycine sublimation conditions were kept constant $\left(T_{\text {oven }}=105^{\circ} \mathrm{C}\right)$.

\section{Polar environments}

Although obscured by the strong water absorption at $\sim 1650 \mathrm{~cm}^{-1}$, panel (b) shows that the glycine spectrum of the water mixture does not change appreciably with respect to that of its pure form. The glycine bands appear at practically the same frequencies and the fraction neutral/zwitterionic remains almost the same. Table 3 collects observed and calculated values of the vibrational modes of neutral glycine (and of its dimer, see below). Our observations agree with those of Gómez-Zavaglia and Fausto ${ }^{26}$ for vapour deposited pure glycine films at $9 \mathrm{~K}$. The spectral evidence points to the conclusion that at low temperature the glycine molecules surrounded by a water ice matrix adopt a configuration that does not differ appreciably from the one it has in vapour deposited pure glycine films. The transformation from

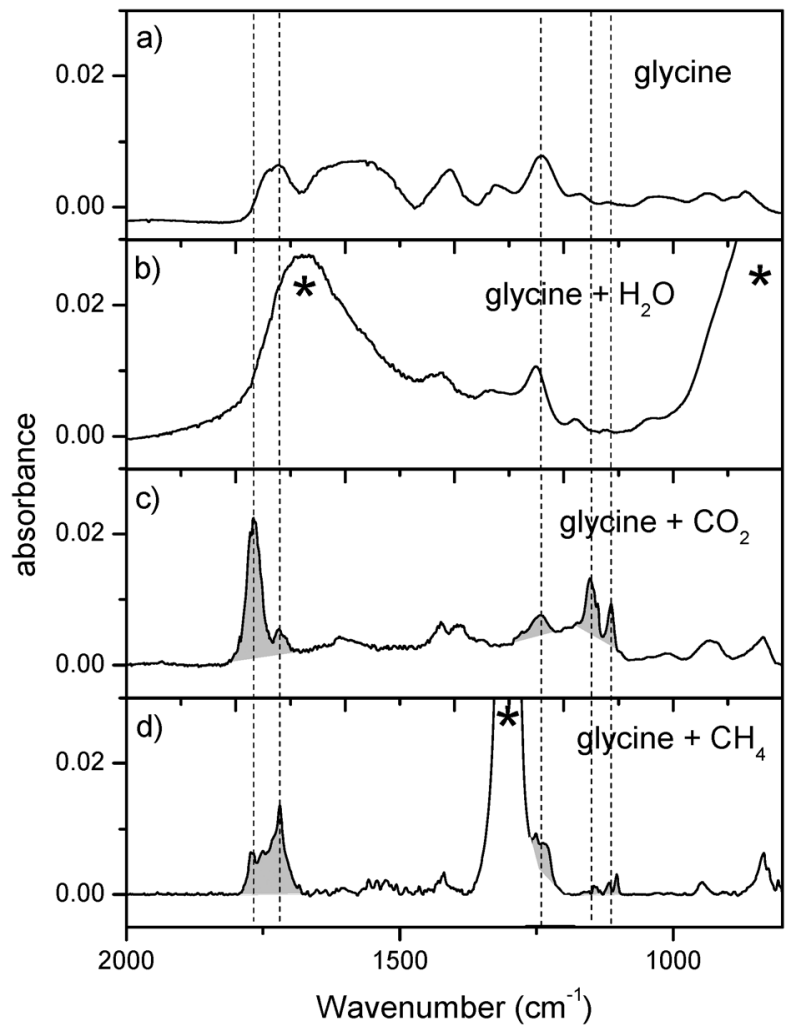

Fig. 5 Infrared spectra of solid layers generated by vapour deposition at $25 \mathrm{~K}$ of (a) glycine, (b) $0.5 \%$ glycine $/ \mathrm{H}_{2} \mathrm{O}$, (c) $0.5 \%$ glycine $/ \mathrm{CO}_{2}$, (d) $0.5 \%$ glycine $/ \mathrm{CH}_{4}$. The asterisks $(*)$ denote $\mathrm{H}_{2} \mathrm{O}$ bands in panel (b), and a $\mathrm{CH}_{4}$ band in panel (d). The shaded bands in panels (c) and (d) are discussed in the text.

neutral to zwitterionic also takes place in a similar fraction in both media. A theoretical study on neutral and zwitterionic glycine $/ \mathrm{H}_{2} \mathrm{O}$ complexes in Ar matrices indicates that the proton transfer between the $\mathrm{NH}_{2}$ and $\mathrm{COOH}$ groups of glycine occurs when the amino acid is $\mathrm{H}$-bonded with at least three water molecules. ${ }^{43}$ In our co-deposited $0.5 \%$ glycine/water samples at $25 \mathrm{~K}$ each glycine molecule must be surrounded by more than three water molecules, and consequently the detected presence of IR absorptions associated to both forms was to be expected.

As we mentioned above, the main fingerprints of the presence of neutral glycine in our solid deposits are the bands at 1725 and $1242 \mathrm{~cm}^{-1}$ in the spectra of Fig. 2, listed in the second column of Table 3. The former is present in the $\mathrm{Ar}$ matrix spectrum ${ }^{18}$ (column 7 th of Table 3 ), but the second one is missing. Gómez-Zavaglia and Fausto ${ }^{26}$ suggested that these bands could be associated to neutral glycine dimers, based on an $a b$ initio calculation of the infrared spectrum of the most stable conformation in the gas phase of these dimers (a centrosymmetric structure with two equivalent $\mathrm{H}$-bonds between carboxyl groups), that predicted strong absorptions around these wavenumbers. Our theoretical calculation for this dimer structure also predicted strong absorptions in these regions, as listed in the last column of Table 3 . However, the relative stability of the different glycine dimer conformers is nowadays a subject of debate (see e.g. ref. 44-46). These studies show that different conformations could present similar stabilization energies, and therefore several conformers of the dimer having 
Table 3 Spectra assignment of neutral glycine in different environments

\begin{tabular}{|c|c|c|c|c|c|c|c|c|c|}
\hline \multirow[b]{3}{*}{ Mode } & \multicolumn{6}{|c|}{ Experimental } & \multicolumn{3}{|l|}{ Theoretical } \\
\hline & \multicolumn{4}{|c|}{ This work $^{a}$} & \multirow[b]{2}{*}{$\begin{array}{l}\text { (Ref. 26) } \\
\text { Solid }\end{array}$} & \multirow[b]{2}{*}{$\begin{array}{l}\text { (Ref. 18) } \\
\text { Ar Matrix }\end{array}$} & \multicolumn{3}{|l|}{ This work $^{b}$} \\
\hline & Solid & $\begin{array}{l}0.5 \% \text { gly } \\
\mathrm{H}_{2} \mathrm{O}\end{array}$ & $\begin{array}{l}0.5 \% \text { gly } \\
\mathrm{CO}_{2}\end{array}$ & $\begin{array}{l}0.5 \% \text { gly } \\
\mathrm{CH}_{4}\end{array}$ & & & $\begin{array}{l}\text { Monomer } \\
\text { (gas phase) }\end{array}$ & $\begin{array}{l}\text { Monomer } \\
\text { (in water) }\end{array}$ & Dimer \\
\hline$\nu \mathrm{C}=\mathrm{O}$ & 1725 & Hidden & 1767,1720 & 1770,1720 & 1715 & 1779 & $1811(297)$ & $1743(600)$ & $1751(701)$ \\
\hline$\delta \mathrm{NH}_{2}$ & 1650 & Hidden & - & - & 1643 & 1630 & 1675 (19) & $1632(46)$ & $1676(31)$ \\
\hline$\delta \mathrm{CH}_{2}$ & 1438 & 1423 & 1423 & 1420 & - & 1429 & 1457 (16) & $1442(31)$ & $\begin{array}{l}1462(43), 1453 \\
(56)\end{array}$ \\
\hline$\nu \mathrm{C}-\mathrm{C}$ & 1326 & 1326 & 1393 & - & - & 1390 & $1395(12)$ & $1395(30)$ & $1376(28)$ \\
\hline$\nu \mathrm{CO}+\delta \mathrm{COH}$ & 1242 & 1250 & 1240 & 1240 & 1247 & - & 1305 (14) & $1291(58)$ & $1279(420)$ \\
\hline$\tau \mathrm{CH}_{2}$ & 1170 & 1175 & 1150 & 1141 & 1157 & 1130 & $1156(87)$ & 1154 (141) & $1139(42)$ \\
\hline$\nu \mathrm{CN}$ & 1117 & 1126 & 1115 & 1117,1106 & - & 1101,1099 & $1117(222)$ & $1112(350)$ & - \\
\hline$\tau \mathrm{CO}$ & 1002 & 1041 & 1013 & - & 1010 & - & - & - & $1009(152)$ \\
\hline$\nu \mathrm{CC}+\omega \mathrm{NH}_{2}$ & 934 & Hidden & 933 & 946 & 938 & 883 & 907 (111) & 908 (141) & 914 (136) \\
\hline$\omega \mathrm{NH}_{2}+\nu \mathrm{CC}$ & 868 & Hidden & 837 & 833 & 866 & 801,798 & $816(89)$ & 819 (167) & $844(321)$ \\
\hline
\end{tabular}

Ref. 26: Pure glycine vapour deposited on a cold substrate at $9 \mathrm{~K}$. Ref. 18: $0.001 \%$ Glycine in an Ar matrix at $13 \mathrm{~K} .{ }^{a}$ Solids generated by vapour deposition on a cold substrate at $25 \mathrm{~K} .{ }^{b}$ Gaussian calculations for a gas phase neutral glycine molecule and dimer, and a neutral glycine monomer in PCM of water.

different infrared spectra could be present in the gas phase. Even more, in a recent paper, ${ }^{45}$ the authors calculated several conformations of glycine dimers, including neutral, zwitterionic and even ionic species, showing that their relative stabilities depend on their environments, with the zwitterionic forms being more stable than the neutral ones in polar solutions. Consequently, the presence of dimers or aggregates of glycine in our deposits cannot be discarded, but for the assignment it must be kept in mind that different conformations could exist, with diverse infrared spectral characteristics.

On the other hand, our calculations of a neutral glycine monomer in water solution (see Table 3) predict infrared bands at 1743 and $1291 \mathrm{~cm}^{-1}$, which agree nicely with those observed in the spectra. These bands become significantly more intense in solution than in the gas phase. According to this result, the presence of these bands in our spectra could simply be rationalized as arising from neutral glycine molecules in a polar environment, created either by zwitterionic glycine or water, or both.

\section{Non-polar environments}

Significant changes are noticeable in the spectra of $0.5 \%$ glycine $/ \mathrm{CO}_{2}$ and glycine $/ \mathrm{CH}_{4}$ layers (panels (c) and (d) in Fig. 5) with respect to the spectrum of the pure form (panel a). The accompanying molecules $\left(\mathrm{CO}_{2}, \mathrm{CH}_{4}\right)$ are less polar than $\mathrm{H}_{2} \mathrm{O}$ or glycine itself, and the conversion from neutral to zwitterionic is highly restricted. Although some zwitterionic form might be present in the glycine $/ \mathrm{CO}_{2}$ or glycine $/ \mathrm{CH}_{4}$ solid layers at $25 \mathrm{~K}$, it is below the sensitivity limit of our IR spectra. We have assigned all the glycine bands observed in these mixtures to the neutral form (isolated or forming aggregates), with the assignments listed in Table 3 . There are also interesting differences between the spectra in the two non-polar environments, being furthermore at variance with the spectrum recorded by Stepanian and coworkers ${ }^{18}$ for neutral glycine in an argon matrix at $13 \mathrm{~K}$. The spectral differences must reflect the diverse interactions that the glycine molecules feel in the vicinity of $\mathrm{CO}_{2}, \mathrm{CH}_{4}$ or Ar atoms in the matrix. We have drawn broken vertical lines to emphasize the largest dissimilarities in the $800-2000 \mathrm{~cm}^{-1}$ mid-IR zone in Fig. 5, where the bands of interest are shaded, and for the $3400-3900 \mathrm{~cm}^{-1} \mathrm{O}-\mathrm{H}$ stretching band region in Fig. 6. The relatively narrow bands at $1150 \mathrm{~cm}^{-1}$ and $1115 \mathrm{~cm}^{-1}$, fairly intense in the $\mathrm{CO}_{2}$ mixture, are assigned to $\mathrm{CH}_{2}$ twisting and $\mathrm{CN}$ stretching, respectively (Gómez-Zavaglia and Fausto's calculation ${ }^{26}$ and also this work), whereas the $1240 \mathrm{~cm}^{-1}$ feature, much more intense in pure glycine and in glycine $/ \mathrm{H}_{2} \mathrm{O}$, is ascribed to the single bond $\mathrm{C}-\mathrm{O}$ stretching in coincidence with a $\mathrm{COH}$ bending. The intensity enhancement in the $1150 \mathrm{~cm}^{-1}$ band for the $\mathrm{CO}_{2}$ mixture could be interpreted in terms of dipole-dipole interactions between the $\mathrm{CH}_{2}$ motion and neighbouring $\mathrm{O}$ atoms of $\mathrm{CO}_{2}$; this interaction would be much reduced for the $\mathrm{CH}_{4}$ sample, where the neighbouring atoms are hydrogen atoms. The opposite would apply to the $1240 \mathrm{~cm}^{-1} \mathrm{C}-\mathrm{O}$ stretching, which would be enhanced in $\mathrm{H}$-rich environments. The intensity of the double bond $\mathrm{C}=\mathrm{O}$ stretch, at $1720 \mathrm{~cm}^{-1}$, seems to be irregularly distributed in the non-polar samples. A very strong band appears in the $\mathrm{CO}_{2}$-dominated solid at $1770 \mathrm{~cm}^{-1}$ accompanied by a weak feature at $1720 \mathrm{~cm}^{-1}$, whereas for the $\mathrm{CH}_{4}$

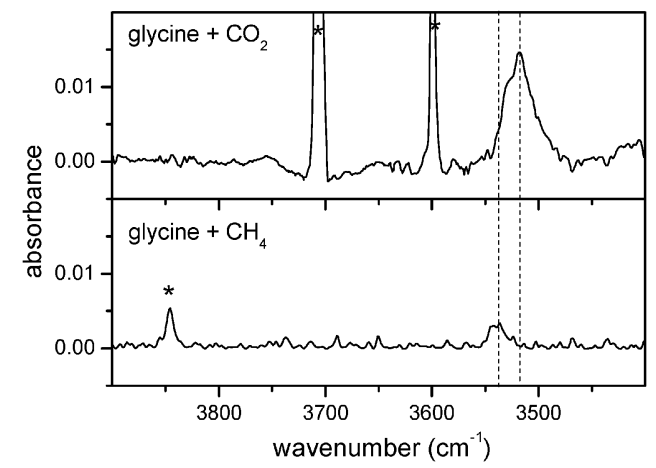

Fig. 6 Detail of the glycine $\mathrm{OH}$ stretching spectral region for the same two mixtures presented in panels (c) and (d) of Fig. 5. Asterisks indicate absorptions of $\mathrm{CO}_{2}$ and of $\mathrm{CH}_{4}$ in the top and bottom panels, respectively. 
case the lower frequency peak is stronger. Without a proper knowledge of the structure of these solids, it is difficult to provide a comprehensive interpretation of these spectral features, in which the interactions between the neutral glycine with different sites of the non-polar surrounding solid must play some role.

It has also been possible to detect the $\mathrm{OH}$ stretching vibration of glycine trapped in these two $\mathrm{CO}_{2}$ and $\mathrm{CH}_{4}$ matrices (in the $\mathrm{H}_{2} \mathrm{O}$ matrix it was buried underneath the $\mathrm{OH}$ stretching bands of water). This region is presented in Fig. 6. The glycine $\mathrm{O}-\mathrm{H}$ absorption band appears at $3520 \mathrm{~cm}^{-1}$ and at $3533 \mathrm{~cm}^{-1}$ for $\mathrm{CO}_{2}$ and $\mathrm{CH}_{4}$ matrices respectively, being in the former spectrum much more intense than in the latter. This vibration is shifted to lower frequencies with respect to the experimental measurements obtained by Huisken et al. ${ }^{47}$ for glycine trapped in $\mathrm{Ar}$ or $\mathrm{Ne}$ matrices, or in He clusters (3566 $\mathrm{cm}^{-1}, 3581 \mathrm{~cm}^{-1}$, and $3585 \mathrm{~cm}^{-1}$ respectively). These authors observed a correlation between the frequency shift of this band and the strength of the interaction with the environment. This rule would imply in our case a stronger interaction with $\mathrm{CO}_{2}$ or $\mathrm{CH}_{4}$ than with the noble gas atoms of the matrices, and between $\mathrm{CO}_{2}$ and $\mathrm{CH}_{4}$, a slightly larger interaction (larger red shift) with the former species. This is also in agreement with the observation of this band being stronger in the $\mathrm{CO}_{2}$ sample than in the methane counterpart, and with the parallel intensity increase in the $1150 \mathrm{~cm}^{-1}$ band in the $\mathrm{CO}_{2}$ sample mentioned above.

Fig. 7 displays the temperature evolution of a pure vapour deposited glycine solid and of the three mixtures studied in this work, all with $0.5 \%$ glycine concentration. For pure glycine (top panel) at the deposition temperature $(25 \mathrm{~K})$, besides the predominant zwitterionic species, we have an important fraction of neutral glycine, revealed by the intensity of the already familiar two main bands associated to this species (shaded in the lowest trace of this panel). The evolution of the intensity of these two bands with temperature, extracted from the spectra shown in the four panels of Fig. 7, is displayed in panels $\mathrm{a}$ and $\mathrm{b}$ of Fig. 8. In the last panel of Fig. 8 a quantization of the fraction of the neutral species present in each deposit, obtained as was explained in the experimental part, is represented. For pure glycine and glycine/water deposits (squares and circles in Fig. 8) a slow decrease of the amount of neutral glycine as the temperature increases is observed. Interestingly, the evolution with temperature of the neutral versus zwitterionic ratio for glycine in a water ice environment is almost the same than in pure glycine, with somehow a little more neutral form in the ice water mixture. It could be expected that the polar water environment might accelerate proton transfer from the neutral to the zwitterion, but the figure indicates a similar pace in both deposits. The two pure neutral glycine bands disappear completely only at $150 \mathrm{~K}$, at the same temperature when glycine is surrounded by other glycine molecules than when it is surrounded by water. At $200 \mathrm{~K}$ most of the water has been evaporated from the sample, and the solid zwitterionic glycine remains.

In $\mathrm{CO}_{2}$ and $\mathrm{CH}_{4}$ environments at $25 \mathrm{~K}$ all the glycine is in its neutral form. Upon heating the transformation to the zwitterionic species takes place very slowly as indicated by the reduced intensity decrease of the stretching $\mathrm{C}=\mathrm{O}$ band at $1770 \mathrm{~cm}^{-1}-1720 \mathrm{~cm}^{-1}$ (see panel a in Fig. 8). When the solids

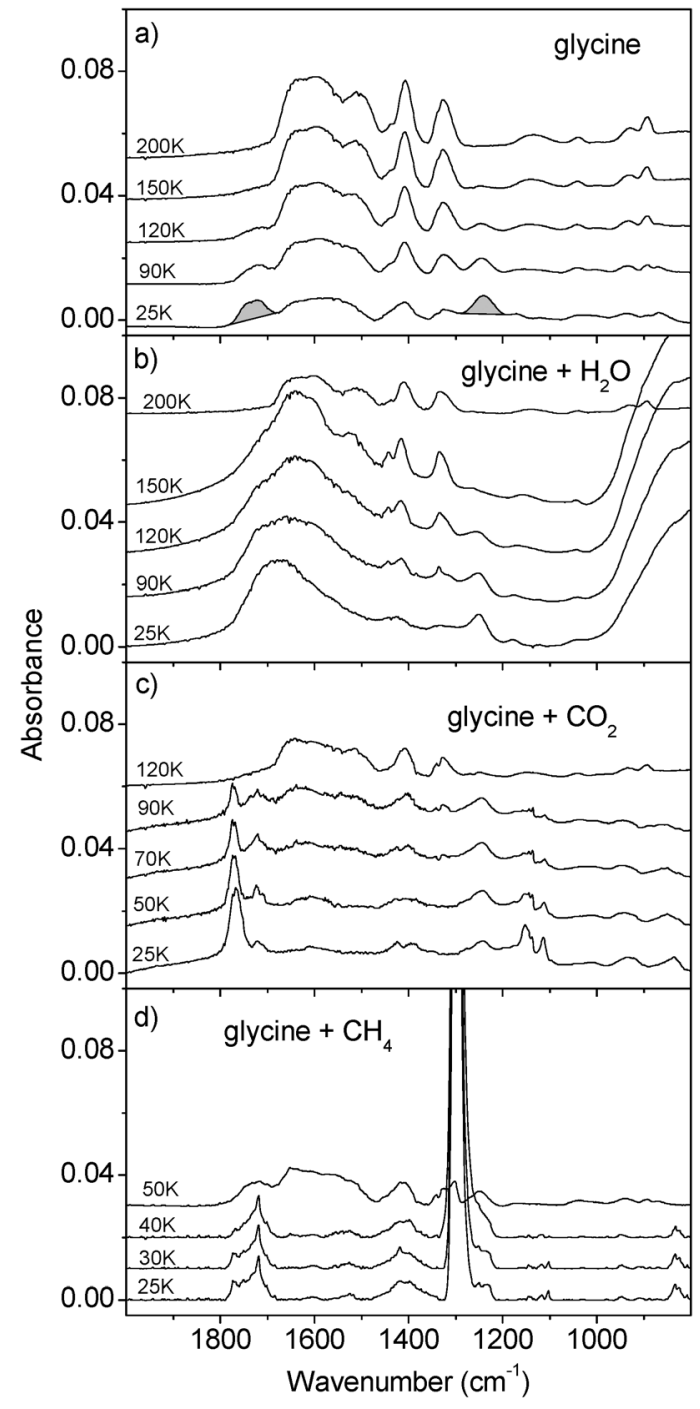

Fig. 7 Temperature evolution of IR spectra of: (a) vapour-deposited pure glycine, (b) $0.5 \%$ glycine $/ \mathrm{H}_{2} \mathrm{O}$, (c) $0.5 \%$ glycine $/ \mathrm{CO}_{2}$, and (d) $0.5 \%$ glycine $/ \mathrm{CH}_{4}$. All samples were deposited at $25 \mathrm{~K}$ and heated up to sublimation of the volatile species. Spectra are offset in the absorbance scale for clarity.

are heated above the sublimation temperature of each volatile, $120 \mathrm{~K}$ for $\mathrm{CO}_{2}$ and $50 \mathrm{~K}$ for $\mathrm{CH}_{4}$, most of these molecules leave the sample and a pure glycine deposit is obtained. Spectra were recorded just above the sublimation temperatures, showing appreciable changes with respect to the corresponding previous cooler spectra. Once the matrix formed by the volatile species disappears the glycine residue quickly reaches the neutral/zwitterionic ratio that corresponds to pure glycine at that temperature (see panel c in Fig. 8).

\section{Summary and conclusions}

This work extends previous knowledge on the IR spectrum of glycine, and on the transformation that this molecule can undergo from the neutral to the zwitterionic form. We generated samples of pure glycine and of diluted mixtures with water, carbon dioxide and methane, molecules that could accompany glycine in astrophysical or astronomical media. 


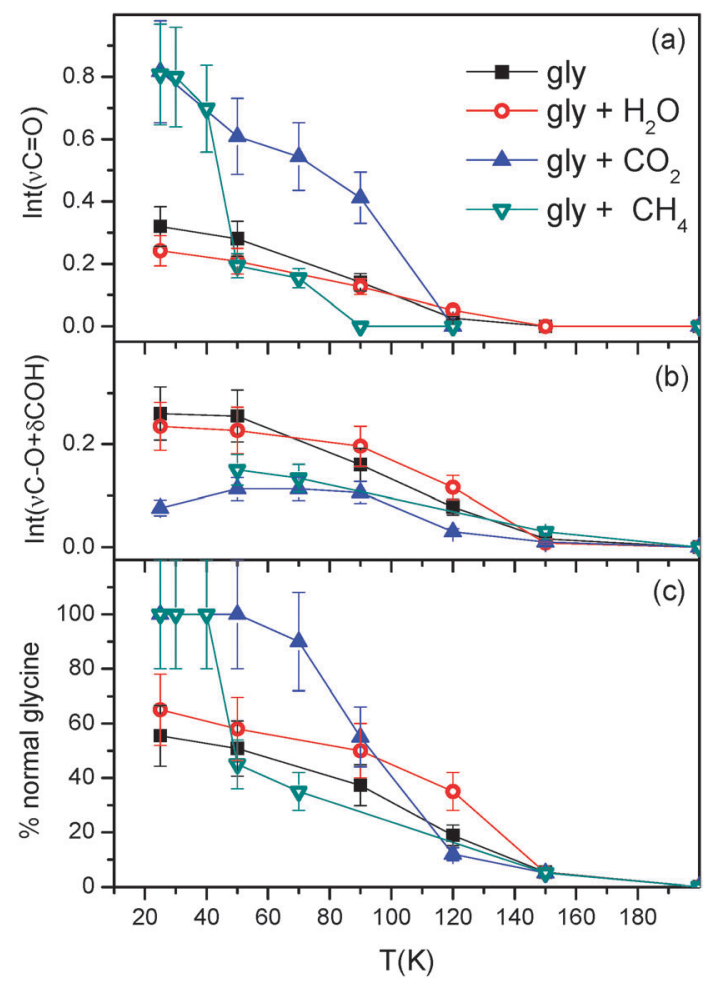

Fig. 8 Panels (a) and (b): temperature evolution of the integrated intensity of the two main IR bands associated to neutral glycine, the $\nu \mathrm{C}=\mathrm{O}$ band at $\sim 1740 \mathrm{~cm}^{-1}$ and the $\nu \mathrm{C}-\mathrm{O}+\delta \mathrm{COH}$ band at $\sim 1420 \mathrm{~cm}^{-1}$, respectively, obtained from the spectra represented in Fig. 7. Panel (c): temperature evolution of the fraction of neutral glycine present in the samples shown in Fig. 7.

Glycine was evaporated in an oven, and the vapour was deposited, together with the other species, on a substrate kept at $25 \mathrm{~K}$ in a low-pressure chamber. The substrate was heated to various temperatures to study the evolution of the substances in the sample using in situ IR spectroscopy. Besides, theoretical calculations were performed on crystalline glycine and on molecular glycine, both isolated and surrounded by water. The main conclusions of this investigation are the following.

Vapour-deposited glycine contains a fraction of neutral glycine that depends on the generation conditions, namely temperature of the oven and of the substrate. Upon heating the neutral form transforms completely to the zwitterionic structure, which is in good agreement with previous observations of room temperature vapour deposited amino acids. ${ }^{15,26}$ The evolution is easily followed by the changes undergone by two IR bands, assigned to the neutral species. This process had been studied before for a $9 \mathrm{~K}$ substrate. ${ }^{26}$ Our results, for a $25 \mathrm{~K}$ substrate, cover a larger range of oven temperatures, and agree with those previously reported.

Diluted mixtures of glycine with water, on one side, and with $\mathrm{CO}_{2}$ and $\mathrm{CH}_{4}$, on the other, show dissimilar characteristics regarding the neutral to zwitterion transformation. In a polar environment $\left(\mathrm{H}_{2} \mathrm{O}\right)$, glycine behaves basically in a similar manner as the pure glycine compound: the neutral content depends on the substrate temperature, decreases with increasing temperature, and is practically null at $150 \mathrm{~K}$. Thus, water or zwitterionic glycine seems to have a similar effect on the neutral glycine molecules in their vicinity, favouring proton transfer to generate the ionic species.

On the other hand, glycine appears only in its neutral form at $25 \mathrm{~K}$ in non-polar surroundings, where the conversion to the zwitterionic structure is highly restricted. As the substrate temperature is raised, the process proceeds very slowly. At the sublimation temperature of the non-polar volatile species the transformation takes place very quickly, and the glycine remains present a very similar pattern to that of pure glycine.

Differences in the spectra recorded at $25 \mathrm{~K}$ for the four systems studied here (pure glycine and three mixtures) are rationalized in terms of dipole interactions between the assigned internal motions for the selected fingerprint vibrations, and the surrounding species. The analysis of the $\mathrm{OH}$ stretching region for the non-polar species indicates a larger interaction between glycine and $\mathrm{CO}_{2}$ than with $\mathrm{CH}_{4}$, and in both cases, stronger interactions than those observed with noble gas matrices too.

A solid state DFT calculation of $\alpha$-glycine yields an optimized structure in reasonable agreement with the neutron diffraction measurements, especially when dispersion corrections are taken into account. The prediction of the IR spectrum of the solid is also in sound concord with the experimental observation. Theoretical calculations at a moderate level, of the individual molecule, the glycine dimer, and the molecule in a water environment offer valuable information that helps in the assignment of the spectra.

From the astrophysical point of view, the main conclusion concerns the possible structure that glycine could have in different media. It seems reasonable to suspect the presence of carbon dioxide and probably methane, besides water, accompanying glycine wherever this species could be found. As mentioned above, these non-polar species favour the neutral form of glycine, and then the most adequate spectral feature to search would be the $1750 \mathrm{~cm}^{-1}(5.71 \mu \mathrm{m})$ band. On the other hand, for warmer samples, or material that has undergone warming above the sublimation temperature of those gases, most glycine would be in its zwitterionic form, and the features to look for should be the $1326 \mathrm{~cm}^{-1}$ and $1423 \mathrm{~cm}^{-1}(7.54 \mu \mathrm{m}$ and $7.03 \mu \mathrm{m}$, respectively) bands, where overlap with the water bending vibration $(6.06 \mu \mathrm{m})$ would be small.

\section{Acknowledgements}

This research has been carried out with funding from the Spanish Ministry of Education, Projects FIS2007-61686 and FIS2010-16455. O.G. and Y.R.-L. acknowledge financial support from Ministerio de Ciencia e Innovación, "Ramón y Cajal" program and CSIC, JAE-Doc Program, respectively. We are grateful to M.A. Moreno, J. Rodríguez and D. Pérez for technical support.

\section{References}

1 J. Oró, Nature, 1961, 190, 389.

2 J. R. Cronin and S. Pizzarello, Adv. Space Res., 1983, 3, 5.

3 S. B. Charnley, et al., Adv. Space Res., 2002, 30(6), 1419.

4 D. Brownlee, et al., Science, 2006, 314, 1711. 
5 S. A. Sandford, et al., Science, 2006, 314, 1720.

6 J. E. Elsila, D. P. Glavin and J. P. Dworkin, et al., Meteorit. Planet. Sci., 2009, 44, 1323.

7 M. Callahan, A. Aubrey, J. L. Bada, J. P. Dworkin, J. E. Elsila, D. P. Glavin, E. Parker and P. Jenniskens, American Astronomical Society, DPS meeting \#41, \#9.09, 09/2009.

8 R. B. Hoover, J. Cosmol, 2011, 13, 3811-3848.

9 G. M. Muñoz Caro, U. J. Meierhenrich and W. A. Schutte, et al., Nature, 2002, 416, 403.

10 M. P. Bernstein, J. P. Dworkin, S. A. Sandford, G. W. Cooper and L. J. Allamandola, Nature, 2002, 416, 401.

11 P. D. Holtom, C. J. Bennett, Y. Osamura, N. J. Mason and R. I. Kaiser, Astrophys. J., 2005, 626, 940.

12 C.-W. Lee, J.-K. Kim, E.-S. Moon, Y. C. Minh and H. Kang, Astrophys. J., 2009, 697, 428.

13 J.-B. Bossa, F. Duvernay, P. Theulé, F. Bogert, L. d'Hendecourt and T. Chiavassa, Astron. Astrophys., 2009, 508(2), 601.

14 M. Nuevo, G. Auger, D. Blanot and L. d'Hendecourt, Origins Life Evol. Biosphere, 2008, 38, 37.

15 U. J. Meierhenrich, J. J. Filippi, C. Meinert, J. B. Bredehöft, J. Takahashi, L. Nahon, N. C. Jones and S. V. Hoffmann, Angew. Chem., Int. Ed., 2010, 49, 7799.

16 F. Duvernay, V. Dufauret, G. Danger, P. Theulé, F. Borget and T. Chiavassa, Astron. Astrophys., 2010, 523, A79.

17 A. Peeters, C. Van Alsenoy, A. T. H. Lenstra and H. J. Geise, J. Chem. Phys., 1995, 103(15), 6608.

18 S. G. Stepanian, I. D. Reva, E. D. Radchenko, M. T. S. Rosado, M. L. T. S. Duarte, R. Fausto and L. Adamowicz, J. Phys. Chem., 1998, 102, 1041.

19 C. Espinoza, J. Szczepanski, M. Vala and N. C. Polfer, J. Phys. Chem. A, 2010, 114, 5919.

20 L. F. Pacios, O. Gálvez and P. C. Gómez, J. Phys. Chem. A, 2001, 105, 5232 .

21 M. T. Rosado, M. L. T. S. Duarte and R. Fausto, Vib. Spectrosc., 1998, 16, 35.

22 R. Ramaekers, J. Pajak, B. Lambie and G. Maes, J. Chem. Phys., 2004, 120(9), 4182.

23 G. Tzvetkov, M. G. Ramsey and F. P. Netzer, J. Chem. Phys., $2005,122,114712$.

24 M. M. Thiam and M. Ebrahimi, e-J. Surf. Sci. Nanotechnol., 2009, 7, 693 .

25 S. Gnanasambandam, Z. Hu, J. Jiang and R. Rajagopalan, J. Phys. Chem. B, 2009, 113, 752 .

26 A. Gómez-Zavaglia and R. Fausto, Phys. Chem. Chem. Phys., $2003,5,3154$.

27 B. Maté, A. Medialdea, M. A. Moreno, R. Escribano and V. J. Herrero, J. Phys. Chem. B, 2003, 107(40), 11098.

28 O. Gálvez, B. Maté, V. J. Herrero and R. Escribano, Icarus, 2008, 197, 599.

29 R. M. Mastrapa, S. A. Shandford, T. L. Roush, D. P. Cruikshank and C. M. D. Ore, Astrophys. J., 2009, 701, 1347.
30 P. A. Gerakines, W. A. Schutte, J. M. Greenberg and E. F. van Dishoeck, Astron. Astrophys., 1995, 296, 810.

31 D. M. Hudgins, S. A. Sandford, L. J. Allamandola and G. G. M. Tielens, Astrophys. J. Suppl., 1993, 86, 713.

32 V. J. Herrero, O. Gálvez, B. Mate and R. Escribano, Phys. Chem. Chem. Phys., 2010, 12, 3164.

33 M. D. Segall, P. J. D. Lindan, M. J. Probert, C. J. Pickard, P. J. Hasnip, S. J. Clark and M. C. Payne, J. Phys.: Condens. Matter, 2002, 14, 2717.

34 J. P. Perdew, K. Burke and M. Ernzerhof, Phys. Rev. Lett., 1996, 77, 3865.

35 J. P. Perdew, J. A. Chevary, S. H. Vosko, K. A. Jackson, M. R. Pederson, D. J. Singh and C. Fiolhais, Phys. Rev. B: Condens. Matter, 1992, 46, 6671.

36 C. Adamo and V. Barony, J. Chem. Phys., 1998, 110, 6158.

37 A. D. Becke, J. Chem. Phys., 1993, 98, 5648.

38 A. Tkatchenko and M. Scheffler, Phys. Rev. Lett., 2009, 102, 73005.

39 S. Grimme, J. Comput. Chem., 2006, 27, 1787.

40 M. J. Frisch, G. W. Trucks, H. B. Schlegel, G. E. Scuseria, M. A. Robb, J. R. Cheeseman, J. A. Montgomery Jr, T. Vreven, K. N. Kudin, J. C. Burant, J. M. Millam, S. S. Iyengar, J. Tomasi, V. Barone, B. Mennucci, M. Cossi, G. Scalmani, N. Rega, G. A. Petersson, H. Nakatsuji, M. Hada, M. Ehara, K. Toyota, R. Fukuda, J. Hasegawa, M. Ishida, T. Nakajima, Y. Honda, O. Kitao, H. Nakai, M. Klene, X. Li, J. E. Knox, H. P. Hratchian, J. B. Cross, V. Bakken, C. Adamo, J. Jaramillo, R. Gomperts, R. E. Stratmann, O. Yazyev, A. J. Austin, R. Cammi, C. Pomelli, J. W. Ochterski, P. Y. Ayala, K. Morokuma, G. A. Voth, P. Salvador, J. J. Dannenberg, V. G. Zakrzewski, S. Dapprich, A. D. Daniels, M. C. Strain, O. Farkas, D. K. Malick, A. D. Rabuck, K. Raghavachari, J. B. Foresman, J. V. Ortiz, Q. Cui, A. G. Baboul, S. Clifford, J. Cioslowski, B. B. Stefanov, G. Liu, A. Liashenko, P. Piskorz, I. Komaromi, R. L. Martin, D. J. Fox, T. Keith, M. A. Al-Laham, C. Y. Peng, A. Nanayakkara, M. Challacombe, P. M. W. Gill, B. Johnson, W. Chen, M. W. Wong, C. Gonzalez and J. A. Pople, Gaussian 03, Gaussian Inc., Wallingford, CT, 2004.

41 P.-G. Jönsson and Å. Kvick, Acta Crystallogr., Sect. B: Struct. Crystallogr. Cryst. Chem., 1972, 28, 1827.

42 O. Gálvez, B. Maté, B. Martín-Llorente, V. J. Herrero and R. Escribano, J. Phys. Chem. A, 2009, 113, 3321.

43 R. Ramaekers, J. Pajak, B. Lambie and G. Maes, J. Chem. Phys., 2004, 120(9), 4182.

44 J. Chocholousova, J. Vacek, F. Huisken, O. Werhahn and P. Hobza, J. Phys. Chem. A, 2002, 106, 11540.

45 M. F. de Carvalho, R. A. Mosquera and R. Rivelino, Chem. Phys. Lett., 2007, 445, 117.

46 P. Friant-Michel and M. F. Ruiz-Lopez, ChemPhysChem, 2010, 11, 3499.

47 F. Huisken, O. Werhahn, A. Y. Ivanov and S. A. Krasnokutski, J. Chem. Phys., 1999, 111(7), 2978. 